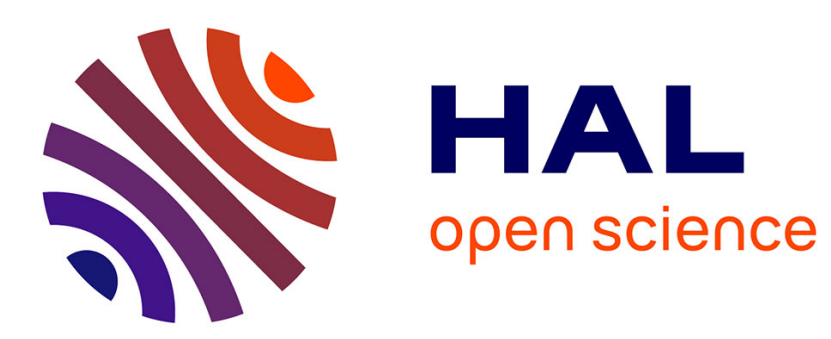

\title{
Mobile Robot Localization Based on a Set Approach using Heterogeneous Measurements
}

\author{
Etienne Colle, Simon Galerne, Maxime Jubert
}

\section{To cite this version:}

Etienne Colle, Simon Galerne, Maxime Jubert. Mobile Robot Localization Based on a Set Approach using Heterogeneous Measurements. 10th International Conference on Informatics in Control, Automation and Robotics (ICINCO 2013), Jul 2013, Reykjavík, Iceland. pp.130-139. hal-00845533

\section{HAL Id: hal-00845533 \\ https://hal.science/hal-00845533}

Submitted on 19 Jul 2013

HAL is a multi-disciplinary open access archive for the deposit and dissemination of scientific research documents, whether they are published or not. The documents may come from teaching and research institutions in France or abroad, or from public or private research centers.
L'archive ouverte pluridisciplinaire HAL, est destinée au dépôt et à la diffusion de documents scientifiques de niveau recherche, publiés ou non, émanant des établissements d'enseignement et de recherche français ou étrangers, des laboratoires publics ou privés. 


\title{
Mobile Robot Localization Based on a Set Approach using Heterogeneous Measurements
}

\author{
Colle, Galerne and Jubert \\ IBISC, University Of Evry, Evry, France \\ \{Etienne.Colle, Simon.Galerne, Maxime.jubert\}@ibisc.univ-evry.fr \\ Corresponding author Etienne.Colle@ibisc.univ-evry.fr
}

\begin{abstract}
Keywords: Robot mobile localization, Interval analysis, Inversion set, Heterogeneous measurements fusion, Cooperative environment

Abstract: This work tackles the problem of the localization of a robot in in large and cooperative environments using real-time data coming either from the robot onboard sensors or/and from the sensors in the environment. The paper focuses on the 3-DOF localization of a mobile robot that is to say the estimation of the robot coordinates $(\mathrm{xmr}, \mathrm{ymr}, \theta \mathrm{mr})$ in a $2 \mathrm{D}$-environment. The problem of nonlinear bounded-error estimation is viewed as a set inversion. The paper presents the theoretical formulation of the localization method in a bounded-error context and the parameter estimation based on interval analysis. Simulation results as well as real experiments show the contributions of the method. The method is able to easily integrate a large variety of sensors, from the roughest to the most complex one. The method takes into account a heterogeneous set of measurements, a flexible number of measurements, a statistical knowledge on the measurements limited to the tolerance, and the fact the measurements are acquired both from the robot onboard sensors and the environment sensors. The way that environment model inaccuracies can be taken into account is also presented.
\end{abstract}

\section{INTRODUCTION}

The ability of a mobile robot to perform various home services for human beings in cluttered or dynamically changing environment requires a reliable robot localization. In the context of the ubiquitous robotics many studies have attempted to improve the accuracy and the reliability of robot localization using the redundancy given by a sensor network. Works are divided into three approaches: localization based on homogeneous sensor network, localization based on hybrid sensor networks and localization based on sensor networks and onboard robot sensors. Zhang and al. [1] exploited a distributed and homogeneous sensor network using infrared sensors. The infrared sensors were suspended from the ceiling. Han and al. [2] proposed a localization system for mobile agents using passive RFID tags that were arranged on the floor of an indoor space. Others authors as Shenoy and al. [3] addressed the localization algorithm with a hybrid sensor network. For accurate localization the existing research might fuse sensor networks and general sensors, Choi [4] [5] proposes a localization scheme based on RFID and a Sonar system. At last robot localization can be based on sensor homogeneous or hybrid sensor networks and onboard robot sensors [6].

The paper addresses the global localization of mobile robots operating in an indoor cooperative environment. The set of home sensors and robot onboard sensors builds a cooperative network robot space. Global localization refers to the problem of estimating the position of a robot (xmr, ymr, $\theta \mathrm{mr})$ in a $2 \mathrm{D}$ reference frame, given the real-time data from the robot onboard sensors and the real-time data coming from sensors located in the environment.

The paper describes a localization method based on interval analysis In the context of bounded-error. The method takes account: i) heterogeneous measurements, ii)a flexible number of measurements, iii) no statistical knowledge about the inaccuracy of measurements, only an admissible interval specified by lower and upper values; he interval is deduced from the sensor tolerance given by manufacturers and iv) measurements both coming from the robot onboard sensors and from the home sensors.

The precise characterization of the measurements errors is conceivable in a laboratory but not at a large scale in the framework of cooperative network space. 
The number and the diversity of sensors are obviously a difficulty for such specific characterization. The errors are usually expressed in terms of stochastic uncertainty models. Due to incomplete information about measurement process, a stochastic error approach is questionable. [7] proposes that the measurement error is no longer considered as a random variable with known probability density function but assumed as bounded between lower and upper values. The set representation is thus poorer but it requires less statistical knowledge on the variables. When the error of measurement on experimental data is known only in the form of a tolerance, which is often the case for the sensors or the network of sensors used in house automation and more generally in the context of ambient intelligence, the set approach is a well-suited approach. On the contrary and moreover if the problem is a linear and Gaussian problem, this approach is not justified because well solved by probabilistic approaches.

The set approach gives a guaranteed result i.e. the solution contains surely the value. The set approach remains little used in the field of mobile robotics. [8] was interested in the localization of a robot starting from measurements of ultrasonic sensors by using the interval analysis and by proposing a treatment of the outliers under certain conditions. [9] uses the interval analysis for modelling inaccurate measurements of two omnidirectional sensors. This work only uses the measurements provided by onboard sensors for robot localization. This idea has been applied by [10] for locating a vehicle with inaccurate telemetric data. More recently, in the field of urban vehicles, works uses various sources of outside or onboard measurement [13]. [11] was interested in multisensor fusion by propagation of constraints on the intervals of measurement provided by the hybridization of a GPS, a gyrometer and an odometer. [12] focused on the robustness of set methods in presence of outliers for multi-sensory localization. Our solution is based on works of [8], more precisely on the algorithm RSIVIA which allows the calculation of solutions by tolerating a number q of outliers. Although the advantages of the probabilistic methods, by far the most used and the best known ones, we have chosen a bounded-error approach based on the interval analysis for the following reasons.

The only assumption to verify is that all the errors are bounded. The respect of this assumption is difficult to prove but there are techniques to reject outliers [14]. If this assumption is verified, then the result is guaranteed. Moreover, as the dimension of the state vector, in our case the $\mathrm{x}$ and $\mathrm{y}$ position and the orientation of the robot, is equal to three, the data processing is relatively simple and fast.

[15] presents a bounded-error state estimation (BESE) to the localization problem of an outdoor vehicle. Authors claim that the biggest advantage of the BESE approach is the ability to solve the localization problem with better consistency than Bayesian approach such as particle filters. Experiments point out that the particle filter can locally converge towards a wrong solution due to bias measurements which lead to a huge local inconsistency. Similar experiments with an Extend Kalman Filter (EKF) show the same phenomenon. EKF strongly underestimates its covariance matrix in presence of repeated biased measurements. The efficiency and accuracy of the particle filter depend mostly on the number of particles. If the imprecision, i.e. bias and noise, in the available data is high, the number of particles needs to be very large in order to obtain good performances. This may give rise to complexity problems for a real-time implementation [16].

The paper is organized as follows. Section 2 describes the principles of the localization method by multiangulation based on interval analysis and set inversion. Section 3 widens the method to heterogeneous measurements not only generic goniometric measurements but also range, the position given by a tactile tile, and dead reckoning measurements. We propose a way to use dead reckoning for data synchronisation. We also explain how to handle environment model inaccuracies. The simulation and experimental results which are respectively described in section 4 and 5 provide information about the accuracy and the computing time of the method.

\section{SET METHOD USING GONIOMETRIC MEASUREMENTS}

The objective of our work is the localization of a mobile robot by using measurements available at a given moment and the a priori known coordinates of the markers or the sensors. The goal is not the building of an environment map but the localization of an assumed-lost robot. The environment is modelled by the coordinates of the home markers seen by the robot onboard sensors and by the coordinates of the home sensors able to detect the 
robot. The markers and the sensors are known by their identifier which makes it possible to establish their location in the building.

The localization process is divided into two steps. The first step consists in finding the room of the building in which the robot is located by using the specific identifier associated to each measure. As said before all sensors and markers are labelled by a specific identifier and associated to one room of the building. The second step localizes the robot inside the room by the set approach described below. The paper focus on this second step.

\subsection{Set Inversion for Estimating Parameters}

Interval analysis [15] is based on the idea of enclosing real numbers in intervals and real vectors in boxes. The analysis by intervals consists in representing the real or integer numbers by intervals which contain them. This idea allowed algorithms whose results are guaranteed, for example for solving a set of non-linear equations [16], [14], [17].

An interval $[x]$ is a set of IR which denotes the set of real interval

$$
[x]=\left\{x \in I R \mid x^{-} \leq x \leq x^{+}, x^{-} \in I R, x^{+} \in I R\right\}
$$

$\mathrm{x}^{-}$and $\mathrm{x}^{+}$are respectively the lower and upper bounds of $[\mathrm{x}]$.

The classical real arithmetic operations can be extended to intervals. Elementary functions also can be extended to intervals.

Given $\mathrm{f}: \mathrm{IR} \rightarrow \mathrm{IR}$, such as $\mathrm{f} \in\{\cos , \sin$, arctan, sqr, sqrt, $\log , \exp , \ldots\}$, its interval inclusion $[\mathrm{f}]([\mathrm{x}])$ is defined on the interval $[\mathrm{x}]$ as follow :

$$
[x] \rightarrow[f]([x])=[\{f(x) \mid x \in[x]\}]
$$

In addition, if $f$ is only composed of continuous operators and functions and if each variable appears at most once in the expression of $f$, then the natural inclusion function of $f$ is minimal. The periodical functions such as trigonometric function require specific treatment. The inclusion function is evaluated by dividing $f$ into a continuous set of monotonic subfunctions.

A subpaving of a box $[\mathbf{x}]$ is the union of nonempty and non-overlapping subboxes of $[\mathbf{x}]$. A guaranteed approximation of a compact set can be bracketed between an inner subpaving $X^{-}$and an outer subpaving $\mathrm{X}^{+}$such as $\mathrm{X}^{-} \subset \mathrm{X} \subset \mathrm{X}^{+}$.

Set inversion is the characterisation of

$$
X=\left\{\mathbf{x} \in I R^{n} \mid \mathbf{f}(\mathbf{x}) \in Y\right\}=\mathbf{f}^{-1}(Y)
$$

For any $\mathrm{Y} \subset \mathrm{IR}^{\mathrm{n}}$ and for any function $\mathbf{f}$ admitting a convergent inclusion function [f], two subpavings $\mathrm{X}^{-}$and $\mathrm{X}^{+}$can be obtained with the algorithm SIVIA (Set Inverter Via Interval Analysis). To check if a box $[\mathbf{x}]$ is inside or outside $\mathrm{X}$,the inclusion test is composed of two tests :

If $[\mathrm{f}]([\mathbf{x}]) \subset \mathrm{Y}$ then $[\mathbf{x}]$ is feasible

If $[\mathrm{f}]([\mathbf{x}]) \cap \mathrm{Y}=\varnothing$ then $[\mathbf{x}]$ is unfeasible

Else $[\mathbf{x}]$ is ambiguous that is feasible, infeasible

Boxes for which these tests failed are bisected except if they are smaller than a required accuracy $\varepsilon$. In this case, boxes remain ambiguous and are added to the $\Delta X$ subpaving of ambiguous boxes. The outer subpaving is $\mathrm{X}^{+}=\mathrm{X}^{-} \cup \Delta \mathrm{X}$. The box is assumed to enclose the solution set $X$.

The inversion set algorithm can be divided into three steps:

- Select the prior feasible box $\left[\mathbf{x}_{0}\right]$ assumed to enclose the solution set $X$;

- Determines the state of a box, feasible, unfeasible or ambiguous;

- Bisect box for reducing $\Delta \mathrm{X}$.

\begin{tabular}{|r|c|}
\hline \multicolumn{2}{|c|}{ Algorithm \#1 SIVIA $\left(\left[\mathbf{x}_{0}\right]\right)$} \\
\hline 1 & if $\left([f]\left(\left[\mathbf{x}_{0}\right]\right) \subset \mathrm{Y}\right),\left[\mathbf{x}_{0}\right]$ is feasible ; \\
\hline 2 & else if $[f]\left(\left[\mathbf{x}_{0}\right]\right) \cap \mathrm{Y}=\varnothing,\left[\mathbf{x}_{0}\right]$ is unfeasible ; \\
\hline 3 & else if $\left(\omega\left(\left[\mathbf{x}_{0}\right]<\varepsilon\right),\left[\mathbf{x}_{0}\right]\right.$ is ambiguous ; \\
\hline 4 & else \\
\hline 5 & bisect $\left.\left[\mathbf{x}_{0}\right],\left[\mathbf{x}_{1}\right],\left[\mathbf{x}_{2}\right]\right) ;$ \\
\hline 6 & SIVIA $\left(\left[\mathbf{x}_{1}\right]\right) ;$ \\
\hline 7 & SIVIA $\left(\left[\mathbf{x}_{2}\right]\right) ;$ \\
\hline 8 & endif \\
\hline 9 & endif \\
\hline 10 & endif \\
\hline
\end{tabular}

This recursive algorithm ends when $\omega[\mathbf{x}]<\varepsilon$. The number $\mathrm{N}$ of bisection is less than

$$
N=\left(\frac{\omega\left(\left|x_{0}\right|\right)}{\varepsilon}+1\right)^{n}
$$

with $\left[\mathbf{x}_{0}\right]$ the prior feasible box and $\mathrm{n}$ the dimension of the vector $[\mathbf{x}]$. Since in the case of the mobile robot localization the dimension of $[\mathbf{x}]$ is three, the solution can be computed with respect to real time. 


\subsection{Application to Localisation by Multiangulation}

The robot localization is computed from several goniometric measurements by multiangulation. Measurements are provided either by robot onboard sensors or/and by home sensors. Onboard robot sensors detect markers located in the environment. Markers can be either RFID tags or visual tags such as Datamatrix, or reference images. On the contrary, what we call home sensors are able to detect the robot and are fixed on a wall, a ceiling or a corner of the rooms. Whatever sensors, the measurement model can be represented by a cone inside which the presence of the robot is guaranteed. This model is simple enough for including a large variety of bearing sensors such presence detector, laser and US telemeters, camera, RFID ...

In the context of bounded-error method, a measurement $\lambda_{\mathrm{i}}$ is defined by an interval bounded by the lower and upper limits:

$$
\left[\lambda_{i}\right]=\left[\lambda_{i}-\Delta \lambda_{i}, \lambda_{i}+\Delta \lambda_{i}\right]
$$

The variables to be estimated are the components of the state vector

$$
\mathbf{x}=\left(\mathrm{x}_{\mathrm{R}}, \mathrm{y}_{\mathrm{R}}, \theta_{\mathrm{R}}\right)^{\mathrm{T}}
$$

which defines the position and orientation of the robot relatively to the reference frame $R_{e}$ of the environment.

The coordinates of the environment markers $M_{j}$ $=\left(\mathrm{x}_{\mathrm{j}}, \mathrm{y}_{\mathrm{j}}\right)$ and the coordinates and orientation of the environment sensors $\mathrm{C}_{\mathrm{j}}=\left(\mathrm{x}_{\mathrm{j}}, \mathrm{y}_{\mathrm{j}}, \theta_{\mathrm{j}}\right)$ are supposed to be known, to be precise coordinate interval is restricted to a scalar value, for sake of readability. However the method we propose can easily take into account inaccuracies on the marker and sensor coordinates.

In our case the problem can be described by two types of equation. In one hand, if a robot sensor detects an environment mark $\mathrm{M}_{\mathrm{i}}$, the measurement depends on the marker coordinates $\mathrm{M}_{\mathrm{i}}\left(\mathrm{x}_{\mathrm{i}}, \mathrm{y}_{\mathrm{i}},\right)$ and the state vector.

$$
\lambda_{i}=\operatorname{tg}^{-1}\left(\frac{y_{R}-y_{i}}{x_{R}-x_{i}}\right)-\theta_{R}
$$

In the other hand (Fig.1b), if the robot is detected by an environment sensor $\mathrm{C}_{\mathrm{i}}$, the measurement depends on the sensor coordinates and orientation $\mathrm{C}_{\mathrm{j}}$ $\left(\mathrm{x}_{\mathrm{j}}, \mathrm{y}_{\mathrm{j}}, \theta_{\mathrm{j}}\right)$ and the state vector.

$$
\lambda_{j}=\operatorname{tg}^{-1}\left(\frac{y_{R}-y_{j}}{x_{R}-x_{j}}\right)-\theta_{j}
$$

The state vector $\mathbf{x}=\left(\mathrm{x}_{\mathrm{R}}, \mathrm{y}_{\mathrm{R}}, \theta_{\mathrm{R}}\right)^{\mathrm{T}}$ is then to be estimated from the $\mathrm{M}$ observations $\lambda=\left(\lambda_{1}, \ldots, \lambda_{\mathrm{M}}\right)$ with the associated bounded errors $[\lambda]=\left(\left[\lambda_{1}\right], \ldots\right.$, $\left.\left[\lambda_{\mathrm{M}}\right]\right)$ and the known data $\mathbf{x}_{\mathrm{i}}=\left(\mathrm{x}_{\mathrm{i}}, \mathrm{y}_{\mathrm{i}}\right)$ and $\mathbf{x}_{\mathrm{j}}=\left(\mathrm{x}_{\mathrm{j}}, \mathrm{y}_{\mathrm{j}}, \theta_{\mathrm{j}}\right)$.

Estimating state vector $\mathbf{x}$ consists in looking for the set $S$ of all admissible values of $\mathbf{x}$ that are consistent with the equations (7) and/or (8) and (5).

In summary, the principal characteristics of the problem are:

- A variable number of nonlinear equations

- $\quad$ Two or three unknown parameters $\left(\mathrm{x}_{\mathrm{R}}, \mathrm{y}_{\mathrm{R}}\right)$ or $\left(\mathrm{x}_{\mathrm{R}}, \mathrm{y}_{\mathrm{R}}, \theta_{\mathrm{R}}\right)$

- A bounded error modelling

- A initial unknown parameter space that can be large

- A Real time constraint (less than one second)

Multiangulation algorithm based on the algorithm SIVIA uses $f(\mathbf{x})=t g^{-1}(\mathbf{x})$ which is a discontinuous function on the interval $[0,2 \pi]$. The estimation of the arctangent inclusion function takes into account both the discontinuities and the border effects due to the fact we manipulate intervals and not values. If we want to consider most of cases, the range of angular measurement can be $\lambda_{\mathrm{i}} \in[0,2 \pi]$ and $\Delta \lambda_{\text {imax }}=\pi / 2$. Indeed, a presence detector can cover an angular sector up to $\pi$ radians.

For each available measure $\lambda_{\mathrm{i}}$, the inclusion test is done using data associated to $\lambda_{\mathrm{i}}$. The test fusion is based on the following rule:

\begin{tabular}{|c|c|}
\hline \multicolumn{2}{|c|}{ Algorithm \# $\quad$ Fusion rule of n inclusion tests } \\
\hline 1 & $\begin{array}{l}\text { if }\left(\mathrm{T}_{1}==\mathrm{T}_{2}==\ldots==\mathrm{T}_{\mathrm{n}}\right), \\
\text { Fusion_test }=\mathrm{T}_{1} ;\end{array}$ \\
\hline 2 & $\begin{array}{l}\text { else if }\left(\left(\mathrm{T}_{1}==\text { unfeasible }\right) \text { or } \ldots \text { or }\right. \\
\left(\mathrm{T}_{\mathrm{n}}==\text { unfeasible }\right), \text { Fusion test }=\text { unfeasible } ;\end{array}$ \\
\hline 3 & else Fusion_test $=$ ambiguous ; \\
\hline 4 & endif \\
\hline 5 & endif \\
\hline
\end{tabular}

This rule leads to reject the result of the algorithm when existing outliers. For processing outliers the fusion rule must to be modified [17]. 


\section{METHOD USING HETEROGENEOUS MEASUREMENTS}

\subsection{Heterogeneous Measurements}

The approach is able to take into account heterogeneous set of measurements. The inclusion test is the same as in the algorithm \#1. It only requires another inclusion function well suited to the measurement type. The right inclusion function is selected thanks to the identifier associated to the sensor. The identifier defines the type of measurement. Combining several measurements is performed by the algorithm \# 2.

The following examples are taken from home automation sensors. Figure 1 shows the features of the three types of measurement with the additional inaccuracy. A ring for goniometric measurement (Fig.1a), a ring and a cone for goniometric and range measurement (Fig.1b) and a square band for tactile tile (Fig.1c).

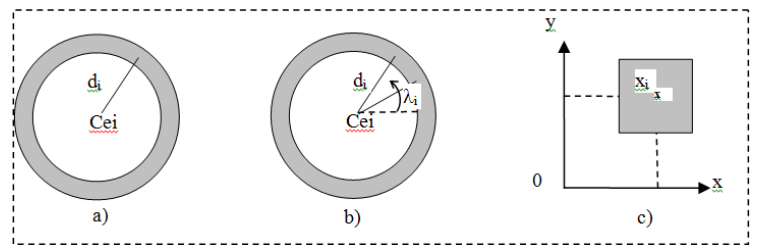

Figure 1: Measurement type: a) Range, b) Goniometric and range, c) Tactile tile

\subsubsection{Goniometric Measurement}

As said in previous section, the measurement is an angle $\lambda_{\mathrm{i}}$ or $\lambda_{\mathrm{j}}$, the measurement model is given either by equation (7) or by equation (8) and the inclusion test is either $[f]\left([\mathbf{x}],\left[\mathbf{x}_{\mathbf{i}}\right]\right) \subset\left[\lambda_{\mathrm{i}}\right]$ or $[\boldsymbol{f}]([\mathbf{x}]$, $\left.\left[\mathbf{x}_{\mathbf{j}}\right]\right) \subset\left[\lambda_{\mathbf{j}}\right]$ with $\mathbf{x}_{\mathbf{i}}$ the environment sensor coordinates and $\mathbf{x}_{\mathbf{j}}$ the marker coordinates.

\subsubsection{Range Measurement}

The measurement is a range $d_{i}$ (Fig.1a), the measurement model is given by $g(\mathrm{x})$ $=\sqrt{\left(x_{R}-x_{j}\right)^{2}+\left(y_{R}-y_{j}\right)^{2}}$ and the inclusion test is $[\mathbf{g}]\left([\mathbf{x}],\left[\mathbf{x}_{\mathbf{i}}\right]\right) \subset\left[\mathrm{d}_{\mathrm{i}}\right]$.

\subsubsection{Goniometric and Range Measurements}

The sensor is supposed able to measure both the angle $\lambda \mathrm{i}$ or $\lambda \mathrm{j}$ and the range di (Fig.1b), the measurement model is given either by $\left(f_{i}(\mathbf{x})\right.$ or $\left.f_{j}(\mathbf{x})\right)$ and $\boldsymbol{g}(\mathbf{x})$ and the inclusion test is either $[\boldsymbol{f}]\left([\mathbf{x}],\left[\mathbf{x}_{\mathbf{i}}\right]\right)$ $\subset\left[\lambda_{\mathrm{i}}\right]$ or $[\boldsymbol{f}]\left([\mathbf{x}],\left[\mathbf{x}_{\mathbf{j}}\right]\right) \subset\left[\lambda_{\mathrm{j}}\right]$ and $[\boldsymbol{g}]\left([\mathbf{x}],\left[\mathbf{x}_{\mathbf{i}}\right]\right) \subset$ $\left[\mathrm{d}_{\mathrm{i}}\right]$.

\subsubsection{Tactile Tile, Door Crossing Detector and Complex Shape}

The measurement are the coordinates of the center of the tile (Fig.1c), the measurement model is $\mathrm{x}_{\mathrm{i}}=\mathrm{x}_{\mathrm{R}}, \mathrm{y}_{\mathrm{i}}=\mathrm{y}_{\mathrm{R}}$ and the inclusion test is $[\mathbf{x}] \subset\left[\mathrm{x}_{\mathrm{i}}\right]$.

The door crossing detector is a variation on the tile model. It is considered as a narrow tile in which the interval associated to each coordinate $\left[\mathrm{x}_{\mathrm{i}}\right]$ and $\left[\mathrm{y}_{\mathrm{i}}\right]$ is different.

A complex shape can be considered as a set of tactile tiles. The measurements are $\left\{\mathrm{C}_{\mathrm{ei}}\left(\mathrm{x}_{\mathrm{i}}, \mathrm{y}_{\mathrm{i}}\right)\right\}$ for $\mathrm{i}=1$ to $\mathrm{n}$, the measurement model is for $\mathrm{i}=1$ to $\mathrm{n}$, $\mathrm{x}_{\mathrm{i}}=\mathrm{x}_{\mathrm{R}}, \mathrm{y}_{\mathrm{i}}=\mathrm{y}_{\mathrm{R}}$ and the inclusion test is for $\mathrm{i}=1$ to $\mathrm{n}$, $[\mathbf{x}] \subset\left[\mathbf{x}_{\mathbf{i}}\right]$.

The literature offers other examples of measure processing by the set approach for localisation, [18] with GPS data or [15] with dead reckoning data. We propose both ways to process the latter kind of measurement.

\subsubsection{Dead Reckoning}

The first way is the same as in cases presented previously. The measurements are $\Delta \mathrm{x}_{\mathrm{i}}, \Delta \mathrm{y}_{\mathrm{i}}, \Delta \theta_{\mathrm{I}}$, the measurement model is $\mathrm{x}_{\mathrm{Rn}}=\mathrm{x}_{\mathrm{Rn}-1}+\Delta \mathrm{x}_{\mathrm{n}}, \mathrm{y}_{\mathrm{Rn}}=\mathrm{y}_{\mathrm{Rn}-1}+\Delta \mathrm{y}_{\mathrm{n}}$, $\theta_{\mathrm{Rn}}=\theta_{\mathrm{Rn}-1}+\Delta \theta_{\mathrm{n}}$ at time $\mathrm{n}$ and $\mathrm{n}-1$ and the inclusion test is $\left[\mathbf{x}_{\mathbf{n}}\right] \subset\left[\mathbf{x}_{\mathbf{n}-1}\right]+\left[\Delta \mathbf{x}_{\mathbf{n}}\right]$.

\subsection{Measurement Synchronisation}

There is another way of using dead reckoning data. When at a given time there are not enough measurements for an accurate localisation, it is possible to take account $\Delta \mathrm{x}_{\mathrm{i}}, \Delta \mathrm{y}_{\mathrm{i}}, \Delta \theta_{\mathrm{I}}$ for synchronising measurements acquired at different times.

For example let three goniometric measurements $\left[\lambda_{\mathrm{n}-2}\right],\left[\lambda_{\mathrm{n}-1}\right],\left[\lambda_{\mathrm{n}}\right]$ acquired at time $\mathrm{t}_{\mathrm{n}-2}, \mathrm{t}_{\mathrm{n}-1}, \mathrm{t}_{\mathrm{n}}$ and $\left[\mathbf{d} \mathbf{x}_{\mathrm{n}-}\right.$ $\left.{ }_{2}\right],\left[\mathbf{d x}_{\mathbf{n}-1}\right]$ the robot displacement given by odometry between $t_{n-2}, t_{n-1}$ and $t_{n-1}, t_{n}$ respectively. [ $\mathbf{x}$ ] can be computed at time $t_{n}$ taking into account both the three measurements $[\lambda]$ and the two relative robot measurements [dx ] by applying the following inclusion test pour $t_{i}=t_{n-2}$ or $t_{n-1}$. 


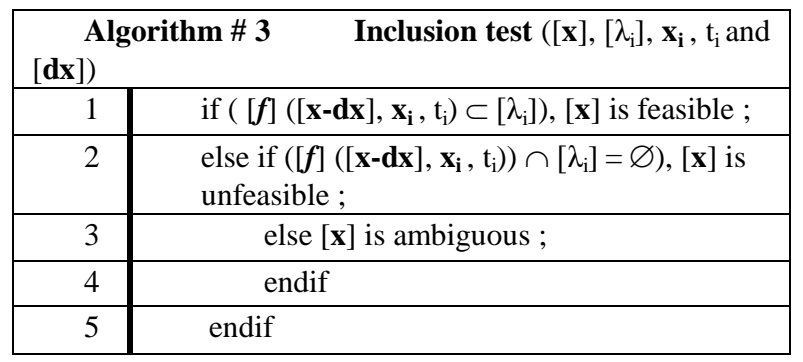

\subsection{Processing of Environment Model Inaccuracies}

The forward-backward contractor method uses a set of variables represented by interval domains with constraints such as equations [19]. All equations or equation systems are available even not invertible ones. Variables may be as well, well-known input variables as unknown output variables, because all variables are processed in the same way. The forward-backward contractor is based on constraint propagation. This contractor makes it possible to contract the domains in order to progress towards the solution and calculate the output variables (note that input variables may be also contracted depending on the measurement tolerance of some input variables); this process is driven by taking into account any one of the constraints, proceeding by intersection of intervals. The aim of propagation technique is to contract as much as possible the domains of the variables without loosing any solution.

Consider $n$ variables $x_{1}, \ldots, x_{n}$ linked by $m$ constraints $\mathrm{C}_{1}, \ldots, \mathrm{C}_{\mathrm{m}}$. For each variable $\mathrm{x}_{\mathrm{i}}$, it is assumed that a prior feasible domain $\left[\mathrm{x}_{\mathrm{i}}\right]=\left[\mathrm{x}_{\mathrm{i}}^{-}, \mathrm{x}_{\mathrm{i}}{ }^{+}\right]$

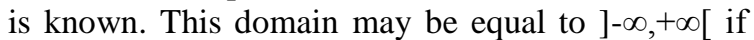
no information is available on $\mathrm{x}_{\mathrm{i}}$. Interval algebra requiring adapted functions, the constraints must be decomposed into primitive constraints, increasing the number of equations and of variables. Then each constraint is calculated according to each variable using interval intersection. Necessarily, the interval length will decrease. This operation is repeated forward and backward until no more significant contraction can be performed. It can be noted that contraction is a quick method which in some cases can slow down or even stop the localisation process before obtaining the desired accuracy. Contraction has to be completed by the bisection method.

This method is able to treat a set of heterogeneous measurement by introducing each measurement equation as a new constraint. We have added a forward-backward contractor step to the bisection algorithm \#2 so as to reduce the solution space and so the computing time.

Moreover the method can reduce the environment model inaccuracies. In our case, we assume that home sensors or markers have been initially approximately located in the home reference frame. The interval domain associated to their coordinates can be reduced with further measurements, either online while detecting outliers, or offline during a learning phase. This is obtained by the typical process of the forward-backward contractor which decreases all interval domains of each variable, input variable as well as output variable.

\section{SIMULATION RESULTS}

The simulation aims at showing: i) the feasibility and the interest of the localization method whatever the position of the sensors and the markers, ii) the ability to integrate a variable number of measurements iii) the ability to mix heterogeneous measurements, iv) the influence of the parameter $\varepsilon$ on the computing time of localization. The algorithm is implemented on Matlab software.

As said in section 2 the indoor localization process is divided into two steps. The first step finds the room in which the robot is located by using the particular identifier associated at each measure. The second step localizes the robot inside the room by the set approach described. The paper dealing with the second step experiments are conducted only in an indoor environment composed of one room.

\subsection{Experimental Protocol}

The robot coordinates are specified in the reference frame. The true measures from the sensors are computed given the known coordinates of the sensors and the markers. Then a specified inaccuracy is added to the measurements in the form of upper and lower bounds.

\subsection{Robot Localisation using Heterogeneous Measurements}

The robot position is represented by two subpavings which include the set of the solution boxes, the feasible subpaving in red (or dark grey) and the ambiguous subpaving in blue/yellow (or light grey). It is necessary to consider both subpavings to guarantee a set containing all possible robot location given the measurements and the noise bounds. 
Figure 2 shows the robot position and orientation $\left(\mathrm{x}_{\mathrm{R}}, \mathrm{y}_{\mathrm{R}}, \theta_{\mathrm{R}}\right)$ using three measurements from the robot onboard sensor which detects three markers labelled $M_{i}$ (Fig.1a). Note that in this case the equations system allows the computing of the robot orientation.

$\begin{array}{ccc}\text { The simulation } & \text { parameters are } \\ {\left[\lambda_{i}\right]=\left[\lambda_{i}-\Delta \lambda_{i}, \lambda_{i}+\Delta \lambda_{i}\right] \quad \text { with } \Delta \lambda_{\mathrm{i}}=\pi / 36,}\end{array}$ $\varepsilon=0.02 \mathrm{~m}$. The true robot configuration is $(4 \mathrm{~m} ; 3 \mathrm{~m}$, $\pi / 4)$.

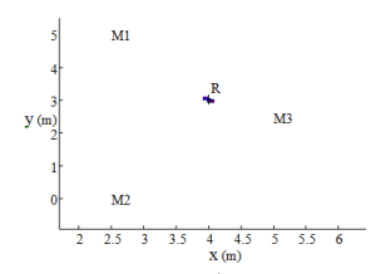

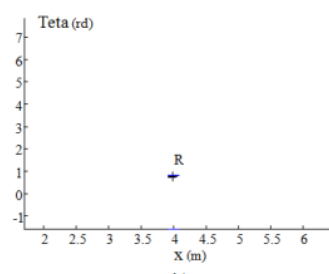

Figure 2 : 2-DOF robot localization a) Projection on the $x$ $\mathrm{y}$ plane, $\mathrm{b}$ ) projection on the $\mathrm{x}-\theta$ plane.

For readability only feasible subpaving is displayed. The results are satisfying in terms of localization accuracy.
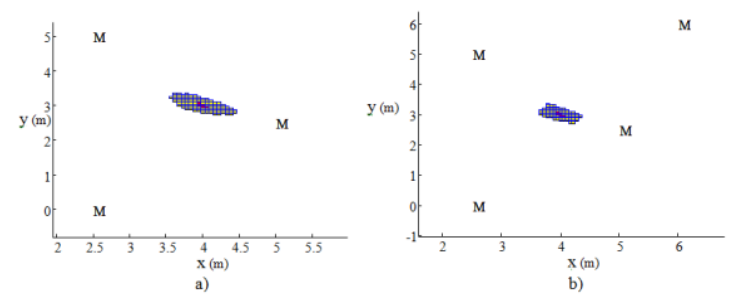

Figure 3: 3-DOF robot localization. Projection in the $x-y$ plane: a) With three available measurements, b) With four available measurements.

One of the interests of the approach is the ability to integrate easily a variable number of measurements. For example if a fourth measurement is available, it is added to other measurements for reducing the localization area (Fig. 3b).
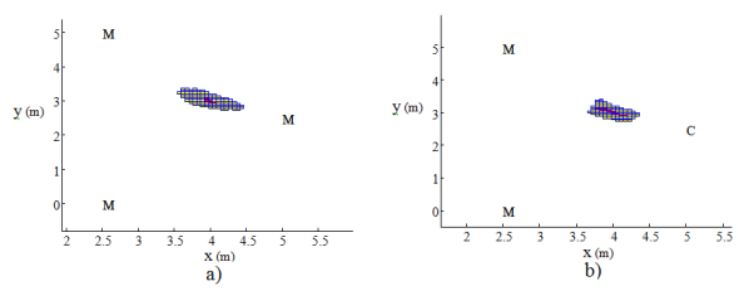

Figure 4: 3-DOF robot localization. Projection in the $x-y$ plane: a) The three measurements are acquired by the robot sensor. b) One of the measurements is acquired by a home sensor $(\mathrm{C})$

The method can without difficulty include both goniometric measurements from onboard robot (M) and from home sensors (C) as illustrated in Figure 4 but also heterogeneous measurements (Fig. 5).

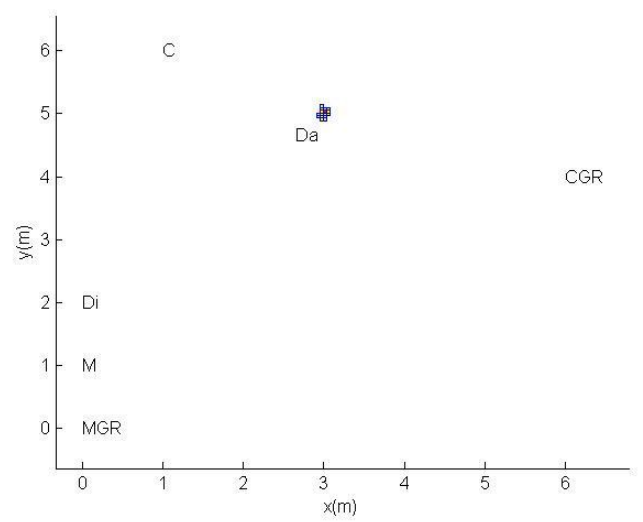

Figure 5: 2-DOF robot localization with 6 heterogeneous measurements.

Label C stands for home goniometric measurement, $\mathrm{M}$ for robot goniometric measurement, Di for range measurement, CGR for home range and goniometric measurement, MGR for robot range and goniometric measurement, Da for tile measurement. The true robot configuration is $(5 ; 3) \mathrm{m}$.

\subsection{Computing Time of Robot Localisation}

In order to verify if the computing time of localization is compatible with the real time constraint of robotic application we have realized two evaluations. The algorithm is implemented on Matlab software.

The first test evaluates the influence of the localization accuracy and of the parameter number on the computing time (Table 1).The simulation parameters are $\left[\lambda_{i}\right\rfloor=\left[\lambda_{i}-\Delta \lambda_{i}, \lambda_{i}+\Delta \lambda_{i}\right\rfloor \quad$ with $\Delta \lambda_{\mathrm{i}}=\pi / 144$, the robot position accuracy $\varepsilon_{\mathrm{xy}}$ varies from $0.5 \mathrm{~m}$ to $0.001 \mathrm{~m}$, the robot orientation accuracy $\varepsilon_{\theta}$ does not change. The room size is $6 \times 6$ $\mathrm{m}^{2}$. The values of table are the mean time for 100 different positions of the robot. The robot orientation does not change, $\theta_{R}=\mathrm{Pi} / 4$. In the first row the 2-dof robot localization is computed from the 
measurements provided by three goniometric sensors located at $(3 ; 0),(0 ; 6),(6 ; 6) \mathrm{m}$. The second row gives the mean time needed for the 3-dof robot localisation. In the latter case the experimental conditions are the same as for the first row. The only difference is that one of the three measurements is necessarily acquired from the robot in order to calculate the robot orientation.

\begin{tabular}{|c|c|c|}
\hline \multirow{2}{*}{ Accuracy $(\mathrm{m})$} & \multicolumn{2}{|c|}{ Computing Time $(\mathrm{s})$} \\
\cline { 2 - 3 } & $\left(\mathrm{x}_{\mathrm{R}}, \mathrm{y}_{\mathrm{R}}\right)$ & $\left(\mathrm{x}_{\mathrm{R}}, \mathrm{y}_{\mathrm{R}}, \theta_{\mathrm{R}}\right)$ \\
\hline 0.5 & 0.01 & 0.24 \\
\hline 0.1 & 0.019 & 0.44 \\
\hline 0.05 & 0.03 & 0.22 \\
\hline 0.025 & 0.05 & 0.22 \\
\hline 0.015 & 0.09 & 1.44 \\
\hline 0.01 & 0.17 & 5.29 \\
\hline 0.001 & 1.24 & \multicolumn{2}{|c}{} \\
\cline { 2 - 3 } & \multicolumn{2}{|c|}{}
\end{tabular}

Table 1: Computing time of the 2-DOF or the 3-DOF robot localization with respect to the localisation accuracy

A 2-dof robot localization can be computed below a second up to $0.01 \mathrm{~m}$ accuracy. A 3-dof robot localization can be computed below a second up to 0.1 accuracy. The robot orientation is time consuming.

The second test evaluates the influence of the number of measurements on the computing time.

The global dimensions of the room are $6 \mathrm{~m} \times 6 \mathrm{~m}$. The localization accuracy is $\varepsilon=0.05 \mathrm{~m}$. The robot coordinates are $X=[3 \mathrm{~m}, 5 \mathrm{~m}, 1 \mathrm{rd}]$. The position and the precision of the sensors are randomly chosen, $\Delta \lambda_{\mathrm{i}} \in[\pi / 72 ; \pi / 72]$. The computing times, mean and standard deviation, are calculated from of 100 random samplings. As explained before one measurement is provided by the robot in order to compute its orientation. We progressively increase the number of sensors. The added sensors are of the same type. The Figure 6 gives a representative example when adding goniometric measurements acquired by home sensors. Whatever the type of sensors added the curve has the same shape.

The second evaluation shows that the computing time depends little on the number of measurements. It is not necessary to develop a strategy for selecting among available measurements. We can take all. It also appears that the standard deviation added to the sampling of the curves decreases with the number of measurements. This fact shows that the computing time is sensor coordinate dependant.

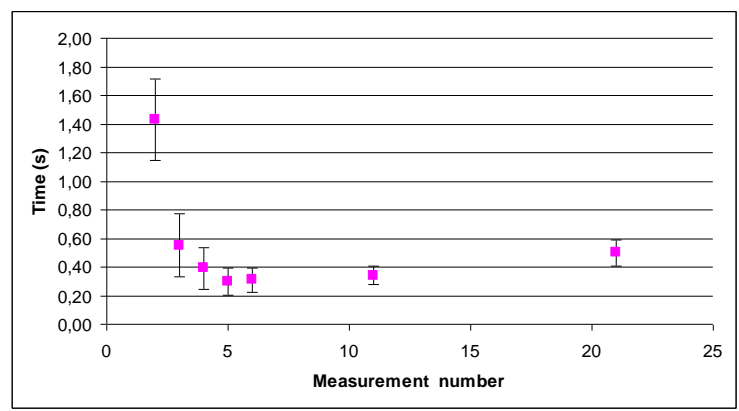

Figure 6: Computing time of the 3-DOF robot localization with respect to the localisation accuracy.

\section{EXPERIMENTAL RESULTS}

Real experiments have been performed with a physical robot in a smart environment composed of two rooms for evaluating the localization method based on interval analysis.

Experiments aim at: i) confirming the simulation results, ii) showing how outliers could be processed and, iii) evaluating the influence of the parameter $\varepsilon$ on the computing time. The algorithm is implemented on Matlab software.

\subsection{Experimental Protocol}

The global dimensions of the test bed are $9.4 \mathrm{~m} \mathrm{x}$ $6.4 \mathrm{~m}$. The rooms are equipped with presence sensors, video cameras fixed on the top of the walls, a pan video camera embarked on the robot and visual markers. The markers located on the walls are detected by the robot video camera. The markers located on the robot are detected by the video cameras fixed on the walls. The Table 2 gives the main characteristics of the test bed sensors. The data of table 2 are used by the algorithm for determining the inclusion function and the upper and lower bounds associated to the measurement. For example the measurement of a presence sensor positioned on the corner will be

$$
\lambda_{j}=\operatorname{tg}^{-1}\left(\frac{y_{R}-0}{x_{R}-6.4}\right)-(p i \times 135 \div 180)
$$

and the lower and upper bounds will be

$$
\left[\lambda_{j}\right]=\left[\lambda_{j}-\left(\frac{p i \times 45}{180}\right), \lambda_{i}+\left(\frac{p i \times 45}{180}\right)\right]
$$

(see section 4). It appears that such a presence sensor covers all the room. 


\begin{tabular}{|c|c|c|c|}
\hline Sensors & $\begin{array}{c}\text { Presence } \\
\text { sensor }\end{array}$ & $\begin{array}{c}\text { Wall } \\
\text { camera }\end{array}$ & $\begin{array}{c}\text { Robot } \\
\text { camera }\end{array}$ \\
\hline Precision $\left(^{\circ}\right)$ & \pm 45 & \pm 5 & \pm 5 \\
\hline Aperture angle $\left(^{\circ}\right)$ & 90 & $\pm 55 / 2$ & $\pm 55 / 2$ \\
\hline $\begin{array}{c}\text { Orientation } \theta_{\mathrm{j}}\left({ }^{\circ}\right) \\
(\text { see Fig1.b) }\end{array}$ & 135 & 225 & $\Theta$ robot \\
\hline $\begin{array}{c}\text { Position }\left(\mathrm{x}_{\mathrm{j}}, \mathrm{y}_{\mathrm{j}}\right)(\mathrm{m}) \\
(\text { see Fig1.b) }\end{array}$ & $(6.4,0)$ & $(3,2.20)$ & $\begin{array}{c}\text { (x robot, } \\
\text { y robot) }\end{array}$ \\
\hline
\end{tabular}

Table 2: Characteristics of the test bed sensors.

The robot is positioned at a specified coordinates $\left(\mathrm{x}_{\mathrm{R}}, \mathrm{y}_{\mathrm{R}}, \theta_{\mathrm{R}}\right)$. Measurements are collected by a gateway which handles the exchanges between the localisation computer and the smart environment.

\subsection{Results}

The Figure 7 shows the robot position estimated by the method from the measurements provided by two wall cameras $(\mathrm{C} 1, \mathrm{C} 2)$. The feasible subpaving is in red (or dark grey) and the ambiguous subpaving in blue/yellow (or light grey). The true robot position is $(3,3.2) \pm 0.2 \mathrm{~m}$ is depicted by an ellipse.

A third measurement from the robot video camera not only improves the position accuracy but also allows the robot orientation, $\theta_{\mathrm{R}}=3 * \mathrm{pi} / 2$ (Fig.12). C1 and $\mathrm{C} 2$ represent the two wall cameras and M3, the marker detected by the robot video camera.
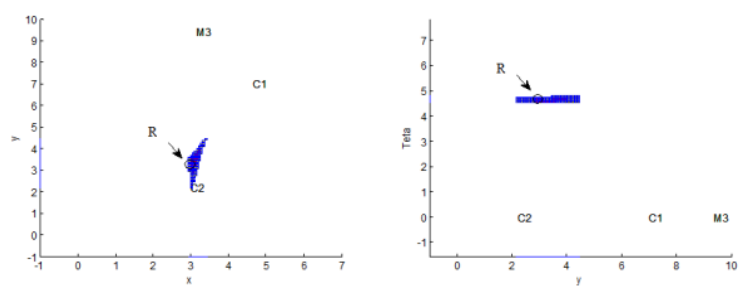

Figure 7: 3-DOF robot localization $(\mathrm{x}, \mathrm{y})$ in meters and $\theta$ in radians. a) Projection in the $x-y$ plane, b) Projection in the $\mathrm{y}-\theta$ plane.

The results of the real experiments are very close of those obtained in simulation. Such results are very useful in poor environment with little sensors because the robot position and orientation are modelled as areas. These areas can be more or less large but it is sure that the robot is inside. Such information is well-suited to topological space representation which is more and more used in robotics in order to simplify databases and be able to treat various qualities of data. It is a promising result easy to improve by using a more efficient programming language.

In order to evaluate a mean computing time for various relative positions of the robot with the sensors $\left(C_{j}\right)$, three home sensors are placed at the vertices of an equilateral triangle (Fig.14). The robot position varies from 1 to 6 meters in $\mathrm{x}$ and $\mathrm{y}$ axis. For each position the set of solution is computed. The result is the 64 robot position map which points up the various form of the subpavings related to the relative positions of the robot with the sensors $\left(\mathrm{C}_{\mathrm{j}}\right)$. The experiment parameters are : $\Delta \lambda_{\mathrm{i}}=\pi / 144$, $\varepsilon=0.05 \mathrm{~m}$.

\subsection{Position Map}

In order to evaluate a mean computing time for various relative positions of the robot with the sensors $\left(C_{j}\right)$, three home sensors are placed at the vertices of an equilateral triangle. Real experiments being more complex to carry out, we have limited the number of robot positions to ten poses. The robot poses are equally distributed on the real environment. Table 4 gives the mean computing time over ten robot poses for three different accuracies $\varepsilon$.

The experiment parameters are: $\Delta \lambda_{i}=\pi / 72$.

\begin{tabular}{|c|c|c|c|}
\hline Epsilon/accuracy (m) & 0.1 & 0.05 & 0.01 \\
\hline $\begin{array}{c}\text { Mean computing time } \\
\text { (s) }\end{array}$ & 0,016 & 0,027 & 0,089 \\
\hline $\begin{array}{c}\text { Mean measurement } \\
\text { frequency (Hz) }\end{array}$ & 61 & 37 & 11 \\
\hline $\begin{array}{c}\text { Mean computing time } \\
\text { (s) given by simulation }\end{array}$ & 0.019 & 0.03 & 0.17 \\
\hline
\end{tabular}

Table 3 : Mean computing time in seconds with respect to the accuracy for ten robot locations.

The results are close to the computing times of the Table 3. As we said before the computing time is relatively stable whatever the robot position in the map relating to the sensors. It is compatible with the real time needs of robotic application even with a Matlab code.

\section{CONCLUSIONS}

The robot localization is based on a interval analysis method applied on data both coming from robot and home sensors. The problem of parameter estimation is solved by a set inversion applied on error bounded 
data. As the parameter vector dimension is two or three, the computing time is compatible with the real time constraint of mobile robotics as showed in sections 4 and 5 . The interest of the solution lies on the ability to integrate a large variety of sensors, from the roughest to the most complex one.

The method is able to take into account i) a heterogeneous set of measurements, ii) a flexible number of measurements, a statistical knowledge on the measurements limited to the tolerance; the sensor model only considers that the measurement is bounded between the lower and upper limits, iii) the ability to include measurements both coming from the robot onboard sensors and from the home sensors.

The algorithm is able to provide a result of localization as soon as only one measure is available. The results show that the computing time depends little on the number of measurements. It is not necessary to develop a strategy for selecting among available measurements. We can take all the measurements available.

The coordinates of the environment markers $M_{j}$ $=\left(\mathrm{x}_{\mathrm{j}}, \mathrm{y}_{\mathrm{j}}\right)$ and the coordinates and orientation of the environment sensors $\mathrm{C}_{\mathrm{j}}=\left(\mathrm{x}_{\mathrm{j}}, \mathrm{y}_{\mathrm{j}}, \theta_{\mathrm{j}}\right)$ are supposed known for paper readability. However the method we propose can easily take into account inaccuracies on the marker and sensor coordinates. We also explain how to handle environment model inaccuracies.

Works in progress address the case where the assumption of bounded error is not verified. The approaches proposed in the literature for processing outliers have to be improved in order to solve all the cases.

\section{REFERENCES}

[1] Z. Zhang, X. Gao, J. Biswas and J. K. Wu, (2007), Moving Targets Detection and Localization in Passive Infrared Sensor Networks, Proceedings of the 10th International Conference on Information Fusion, Quebec.

[2] S. Han, H. Lim and J. Lee, (2007), An Efficient Localization Scheme for a Differential-Driving Mobile Robot Based on RFID System," IEEE Transaction on Industrial Electronics, Vol. 6, 3362-3369.

[3] S. Shenoy and J. Tan, (2005), Simultaneous Localization and Mobile Robot Navigation in a Hybrid Sensor Network, Proceedings of IEEE/RSJ International Conference on Intelligent Robots and Systems, Alberta.

[4] B.-S. Choi and J.-J. Lee, (2009), Mobile Robot Localization Scheme Based on RFID and Sonar Fusion System, Proceedings of IEEE International Symposium on Industrial Electronics, Seoul, 1035-1040.
[5] B.-S. Choi and J.-J. Lee, (2010), Sensor Network Based Localization Algorithm using Fusion Sensor-Agent for Indoor Service Robot, IEEE Transaction on Consumer Electronics, Vol. 56, No. 3, 1457-1465.

[6] Corominas Murtra, A., Mirats Tur, J.M., Sanfeliu, A. (2008). Action Evaluation for Mobile Robot Global Localization in Cooperative Environments, Journal of Robotics and Autonomous Systems, Special Issue on Network Robot Systems.

[7] S. Brahim-Belhouari, M. Kieffer, G. Fleury, L. Jaulin and E. Walter, (2000), Model selection via worstcase criterion for nonlinear bounded-error estimation, IEEE Instrumentation and Measurement Vol. 49, No 3, 653-658.

[8] L. Jaulin, M. Kieffer, E. Walter, and D. Meizel, (2002), Guaranteed Robust Nonlinear Estimation With Application to Robot Localization, IEEE Trans. SMC, PartC Applications and Review, Vol. 32 , No 4, 254-267.

[9] C. Drocourt, (2002). Localization et modélisation de l'environnement d'un robot mobile par coopération de deux capteurs omnidirectionnels, thèse.

[10] O. Lévêque, L. Jaullin, D. Meizel and E. Walter, (1997). Vehicule localization from inaccurate telemetric data: a set of inversion approach. IFAC Symposium on robot Control SYROCO 97, Vol. 1, Nantes, 179-186.

[11] A. Gning, (2006). Fusion multisensorielle ensembliste par propagation de contraintes sur les intervalles, Thèse.

[12] V. Drevelle and P. Bonnifait, (2010), Robust positioning using relaxed constraint-propagation. IROS 2010, Taipei, Vol. 10, 4843-4848.

[13] O. Reynet, L. Jaulin and G. Chabert, (2009), Robust TDOA Passive Location Using Interval Analysis and Contractor Programming, Radar, Bordeaux.

[14] L. Jaulin(2009), Robust set-membership state estimation; application to underwater robotics. Automatica, Vol. 45, No 1, 202-206.

[15] A. Lambert, D. Gruyer, B. Vincke, E. Seignez, (2009), Consistent Outdoor Vehicle Localization by Bounded-Error State Estimation, Intelligent Robots and Systems, IROS , 1211-1216.

[16] Fahed Abdallah, Amadou Gning, Philippe Bonnifait, (2008), Box particle filtering for nonlinear state estimation using interval analysis, Automatica, Vol. 44, No. 3, 807-815.

[15] R.E. Moore, (1979). Method and applications of internal analysis, ed. SIAM, Philadelphia.

[16] L. Jaulin and E. Walter, (1993), Set inversion via interval analysis for nonlinear bounded-error estimation. Automatica, Vol. 29, No 4,1053-1064.

[17] M. Kieffer, L. Jaulin, E. Walter,(2000), D. Meizel, Robust autonomous robot localization using interval analysis, Reliable Computing, Vol. 6, No 3, 337362.

[18] V. Drevelle P. Bonnifait, (2009), ENC-GNSS 2009 European Navigation Conference - Global Navigation Satellite Systems, Naples.

[19] L. Jaulin, M. Kieffer, O. Didrit, and E. Walter, (2001). Applied interval analysis.In Springer-Verlag. 